\title{
Mapping fine-scale anatomy of gray matter, white matter, and trigeminal-root region applying spherical deconvolution to high-resolution 7-T diffusion MRI
}

\author{
Ralf Lützkendorf ${ }^{1} \cdot$ Robin M. Heidemann $^{2}$-Thorsten Feiweier ${ }^{2} \cdot$ Michael Luchtmann $^{3}$ - Sebastian Baecke ${ }^{1}$. \\ Jörn Kaufmann ${ }^{4}$. Jörg Stadler ${ }^{5}$ Eike Budinger ${ }^{5,6}$ • Johannes Bernarding ${ }^{1,6}$
}

Received: 9 December 2017 / Revised: 29 August 2018 / Accepted: 3 September 2018 / Published online: 17 September 2018

(c) The Author(s) 2018

\begin{abstract}
Objectives We assessed the use of high-resolution ultra-high-field diffusion magnetic resonance imaging (dMRI) to determine neuronal fiber orientation density functions (fODFs) throughout the human brain, including gray matter (GM), white matter (WM), and small intertwined structures in the cerebellopontine region.

Materials and methods We acquired 7-T whole-brain dMRI data of 23 volunteers with 1.4-mm isotropic resolution; fODFs were estimated using constrained spherical deconvolution.

Results High-resolution fODFs enabled a detailed view of the intravoxel distributions of fiber populations in the whole brain. In the brainstem region, the fODF of the extra- and intrapontine parts of the trigeminus could be resolved. Intrapontine trigeminal fiber populations were crossed in a network-like fashion by fiber populations of the surrounding cerebellopontine tracts. In cortical GM, additional evidence was found that in parts of primary somatosensory cortex, fODFs seem to be oriented less perpendicular to the cortical surface than in GM of motor, premotor, and secondary somatosensory cortices. Conclusion With 7-T MRI being introduced into clinical routine, high-resolution dMRI and derived measures such as fODFs can serve to characterize fine-scale anatomic structures as a prerequisite to detecting pathologies in GM and small or intertwined WM tracts.
\end{abstract}

Keywords UHF MRI · 7-T · DWI · Trigeminus · Gray matter · fODF

Electronic supplementary material The online version of this article (https://doi.org/10.1007/s10334-018-0705-9) contains supplementary material, which is available to authorized users.

Ralf Lützkendorf

ralf.luetzkendorf@med.ovgu.de

1 Institute for Biometry and Medical Informatics, Otto-vonGuericke-University, Magdeburg, Germany

2 Siemens Healthcare GmbH, Erlangen, Germany

3 Department of Neurosurgery, Otto-von-Guericke-University, Magdeburg, Germany

4 Department of Neurology, Otto-von-Guericke-University, Magdeburg, Germany

5 Leibniz Institute for Neurobiology, Magdeburg, Germany

6 Center of Behavioral Brain Sciences, Magdeburg, Germany

\section{Introduction}

In biological tissue, the mean diffusion length of water protons provides indirect information about the microanatomy of both healthy and metabolically compromised tissue [1-3]. In routine clinical practice, diffusion-weighted magnetic resonance imaging (dMRI) is well established, especially for the early diagnosis of pathologic changes due to ischemic brain stroke [4-8]. Anisotropic diffusion serves to noninvasively characterize white matter (WM) tracts for presurgical planning or scientific research [9-14]. Recently, developments in hardware, data acquisition techniques, and postprocessing algorithms [15-34] have been made, which improved mapping the complex neuronal connections of the human brain. These developments also enabled diffusion characteristics to be mapped not only of WM but even of gray matter (GM) or of small intertwined fiber tracts, as present in the cerebellopontine region. 
After a decade of experimental ultra-high-field MRI 7-T, MR scanners are now being introduced into clinical routine with the expectation that advantages of 7-T, such as increased signal-to-noise ratio (SNR), can help detect, e.g., smaller pathologies. Besides the inner structure of WM tracts in the cerebrum and cerebellum, the brainstem itself and respective entries and exits of the cranial nerves $(\mathrm{CNs})$, in particular, are of high importance for clinicians. Recently, radiation-induced damage to the trigeminal nerve (nerve V) were found using dMRI [35]. High-resolution dMRI is required for characterization of diffusion properties of CNs, especially of the thinner CNs (such as nerves IV, IX, XI, XII), which typically exhibit an extension of only a few millimetres, and of CNs, which have a complex composition of several radixes or subnerves (such as nerves V, VII, VIII, X-XII) or of the intrapontine parts of CNs, which often cross with local brainstem fibers [35-41]. For instance, because intrapontine fibers of the trigeminal nerve exhibit similar relaxation behavior to that of the surrounding pontine fiber tracts, only direction-dependent information as provided by high-resolution dMRI can depict these different fiber distributions and their courses. Recently, Behan et al. [42] provided 3-T dMRI data of ten patients with tumors in the posterior fossa while tracking CNs V and VII/VIII with a resolution of $0.94 \times 0.94 \times 3 \mathrm{~mm}^{3}$ and a diffusion weighting of $b=1000 \mathrm{~s} / \mathrm{mm}^{2}$. The authors focused on comparing three different tracking algorithms - one of them based on constrained spherical deconvolution (CSD)—but did not discuss intrapontine fiber orientation characteristics. Therefore, the first goal of our study was to analyze in more detail the fODF of the trigeminal nerve in a cohort of 23 individuals using CSD [26, 27] of high-resolution 7-T dMRI.

Little is known about diffusion characteristics in GM. Diffusion imaging has been used to monitor maturing brain tissue [43], but in vivo data of human adult GM remains limited [15, 16, 31, 44-48]. Cortical GM also has a more complex microarchitecture than do the relatively larger and often more homogeneous WM tracts. Many microarchitectural features (e.g., number, thickness, layer density) of GM areas vary, as seen in the differences between the primary motor cortex (M1; e.g., nearly absent layer IV) and the primary somatosensory cortex (S1; e.g., thick layer IV) [49]. Thus, our second goal was to investigate fODFs with high resolution not only in the well-known large WM tracts of the brain but also in the cortical and subcortical GM, including the midbrain and cerebellopontine hindbrain regions.

Anwander et al. [45] found evidence that in vivo human GM fibers are more radially oriented (i.e., perpendicular to the complex folded cortical surface) in the GM of M1, whereas in the GM of S1, they are more tangentially oriented to the surface. A recent high-resolution diffusion study with $0.240-\mathrm{mm}$ isotropic resolution of an ex vivo specimen of the primary human visual cortex (V1) even succeeded in distinguishing layer-specific diffusion orientation characteristics [50]. Similar to the results of Anwander et al. [45], in vivo studies by McNab et al. [44] and Calamante et al. [51] reported that M1 fibers are arranged perpendicular to the cortical surface, whereas in parts of S1, diffusion is mainly directed tangential to the cortical surface.

In this study, we performed dMRI of 23 healthy volunteers with $1.4-\mathrm{mm}$ isotropic resolution, which provided a good compromise between SNR and measurement time when acquiring whole-brain data (see our pilot study [52]). To depict fODFs within each voxel, we used CSD, taking into account that both our preliminary work $[16,53,54]$ and the work of other groups [50] showed that this approach provides best results when analyzing diffusion behavior within large- and fine-scale brain structures, including those within GM and across the GM/WM borders.

\section{Materials and methods}

\section{Participants}

Twenty-three healthy adults (ten men, 13 women; mean age $27.6 \pm 4.6$ years) participated in this study after having given their written consent. The study was approved by the Ethics Committee of the Otto-von-Guericke-University Magdeburg in accordance with the principles of the Declaration of Helsinki.

\section{Data acquisition}

All data were acquired on a 7-T whole-body research MRI scanner (Siemens Healthcare, Erlangen, Germany) equipped with a $70-\mathrm{mT} / \mathrm{m}$ gradient coil with a slew rate of $200 \mathrm{~T} / \mathrm{m} / \mathrm{s}$ and using a 32-channel phased-array head coil (Nova Medical, USA). The protocol included a high-resolution anatomical scan [magnetization prepared rapid-acquisition gradient echo (MPRAGE)] 0.8-mm isotropic resolution covering the whole head, including the cerebellum. Diffusion-weighted MRI with 1.4-mm isotropic resolution were acquired from the whole brain in a single session per study volunteer using a prototype single-shot echo-planar imaging (EPI) sequence employing a modified Stejskal Tanner diffusion-encoding gradient scheme [2, 55]. For this study, 128 diffusionweighted data sets $\left(b=1000 \mathrm{~s} / \mathrm{mm}^{2}\right)$ were acquired with different combinations of gradient directions employing the protocol design as per Caruyer (http://www.emmanuelca ruyer.com/q-space-sampling.php) [56, 57]. The acquisition consisted of a cycle of one non-diffusion-weighted data set ( $b_{0}$ map, $b=0 \mathrm{~s} / \mathrm{mm}^{2}$ ), followed by 16 diffusion-weighted data sets $\left(b=1000 \mathrm{~s} / \mathrm{mm}^{2}\right)$. This was repeated until all 128 diffusion directions were sampled. Thus, nine non-diffusion-weighted data sets were acquired to serve for motion 
correction (see below), leading to a total of 137 scans for dMRI data acquisition.

Other imaging parameters were as follows: generalized autocalibrating partial parallel acquisition (GRAPPA) for parallel imaging (acceleration factor 3, partial Fourier acquisition covering 6/8 of k-space), bandwidth $1526 \mathrm{~Hz} /$ pixel, echo spacing $0.76 \mathrm{~ms}, \mathrm{TE}=73 \mathrm{~ms}$, base resolution $156 \times 156$, 98 slices, FOV $220 \times 220 \mathrm{~mm}^{2}$, covering the whole brain, including the cerebellum (measurement time $50 \mathrm{~min}$ ). For comparison of the resolution (see Fig. 1), data of one volunteer were additionally obtained with 2-mm isotropic resolution under otherwise similar technical conditions.

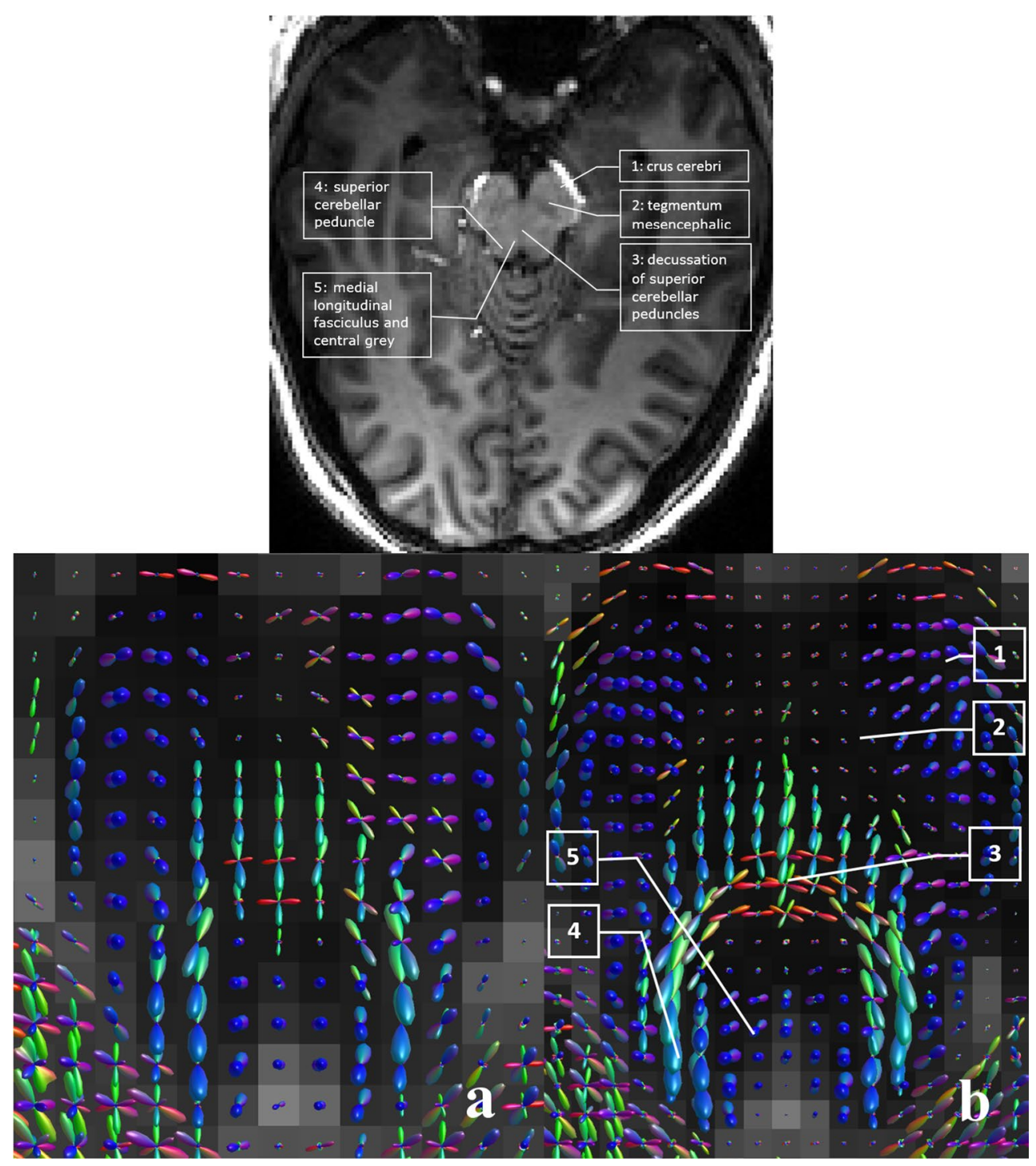

Fig. 1 Impact of resolution on fiber orientation density functions (fODF) in the midbrain region (images displayed in radiological convention, i.e., the left side of the image corresponds to the right side of the patient, the upper half to ventral, and the lower half to dorsal). Top row: anatomic image [magnetization prepared rapid-acquisition gradient echo (MPRAGE), 0.8-mm isotropic resolution, axial orientation] of the midbrain region for identifying the main anatomic structures. Bottom row: fODF maps of the midbrain region derived from dMRI data with 2.0-mm (a) and 1.4-mm (b) isotropic resolution [52]. Data were acquired from the same volunteer; fODF maps were overlaid onto the corresponding non-diffusion-weighted image (i.e., T2-weighted $b_{0}$ map). At $2.0-\mathrm{mm}$ resolution, only a few voxels and the corresponding fiber orientation distribution within these voxels, along with overall fiber bending, are resolved. In contrast, at 1.4-mm isotropic resolution, orientations of the fiber populations can be seen with sufficient detail to distinguish between several fiber bundles crossing and bending in the brainstem ( 1 crus cerebri; 2 tegmentum mesencephalic; 3 decussation of superior cerebellar peduncles; 4 superior cerebellar peduncle; 5 medial longitudinal fasciculus and central grey). Colors of the fODF maps encode directions according to scanner axes left-right ( $x$ direction, red), dorsal-ventral ( $y$ direction, green), and rostrocaudal ( $z$ direction, blue). Mixed colors correspond to nonorthogonal directions. Color encoding is independent from the slice view (axial, coronal, and sagittal). The estimated partial contribution of each fiber direction is represented by the amplitude of the corresponding spherical harmonic function. The direction of the position vector of each surface point is used to assign the color to this point 


\section{Data processing}

Data were postprocessed using FSL (http://fsl.fmrib.ox. ac.uk/fsl/fslwiki) and MRtrix 3.0 (https://www.mrtrix.org) implemented on Ubuntu LINUX (16.04 LTS). Data were corrected for eddy currents with FSL. To account for motion during the measurement, data were processed with functional magnetic resonance imaging of the brain (FMRIB's) linear registration tool (FLIRT) [58] in FSL [59] by registration of the interspersed non-diffusion-weighted images to the first reference (non-diffusion-weighted) image, resulting in eight transformation matrices. Each of the eight transformation matrices was used to realign the corresponding diffusion-weighted data sets, resulting in an overall registration of every data set to the first non-diffusion-weighted data set. The gradient vector scheme had to be adapted separately [57] using a procedure developed in-house and implemented as a UNIX bash script. CSD, diffusion tensors, fractional anisotropy (FA), and eigenvalues of the diffusion tensors were calculated using MRtrix 3.0. The MRtrix module DWI$2 F O D$ was used for estimating the fODF. For optimum visualization of the latter, the threshold was set to 0.16 [27]. The remaining parameters were set to standard values $\left(l_{\max }=8\right.$, neg_lambda $=1$, norm_lambda $=1$; for interpretation of module DWI2FOD and parameters, see https://mrtrix.readt hedocs.io/en/latest/reference/commands/dwi2fod.html) [27].

\section{Results}

Figure 1 shows a representative example illustrating the impact of image resolution on the diffusion information. When compared with the data set acquired with an isotropic resolution of $2 \mathrm{~mm}$ (Fig. 1a), data of the same volunteer acquired with an isotropic voxel size of $1.4 \mathrm{~mm}$ (Fig. 1b) provide many more details about crossing and bending fibers, even if they are densely interwoven (Fig. 1b, no. 3). The fibers leading toward the contralateral side of the midbrain (red) can be clearly identified by their arc-like courses: dorsal fibers bend medially to the central midbrain and traverse to the contralateral dorsal side, where they form the medial part of the superior cerebellar peduncles (Fig. 1b, no. 4). Other fibers from the ipsilateral ventral part of the midbrain (tegmentum, Fig. 1b, no. 2) form additional parts of the cerebellar peduncles (green). The fibers of the medial longitudinal fasciculus are located between the superior cerebellar peduncles (dark blue, Fig. 1b, no. 5). The crura cerebri (comprising the corticospinal and corticopontine fibers; dark blue, Fig. 1b, no. 1), which are vetrolaterally localized, are separated sharply from the anterior-posterior midbrain fiber bundles (green).
Figure 2 displays the anatomically complex pontine region where fibers from the cerebrum and cerebellum (including the cerebral and cerebellar peduncles), from the midbrain and spinal cord and various CNs cross. This imposes high demands on clinicians and neuroimaging researchers when attempting to analyze the corresponding

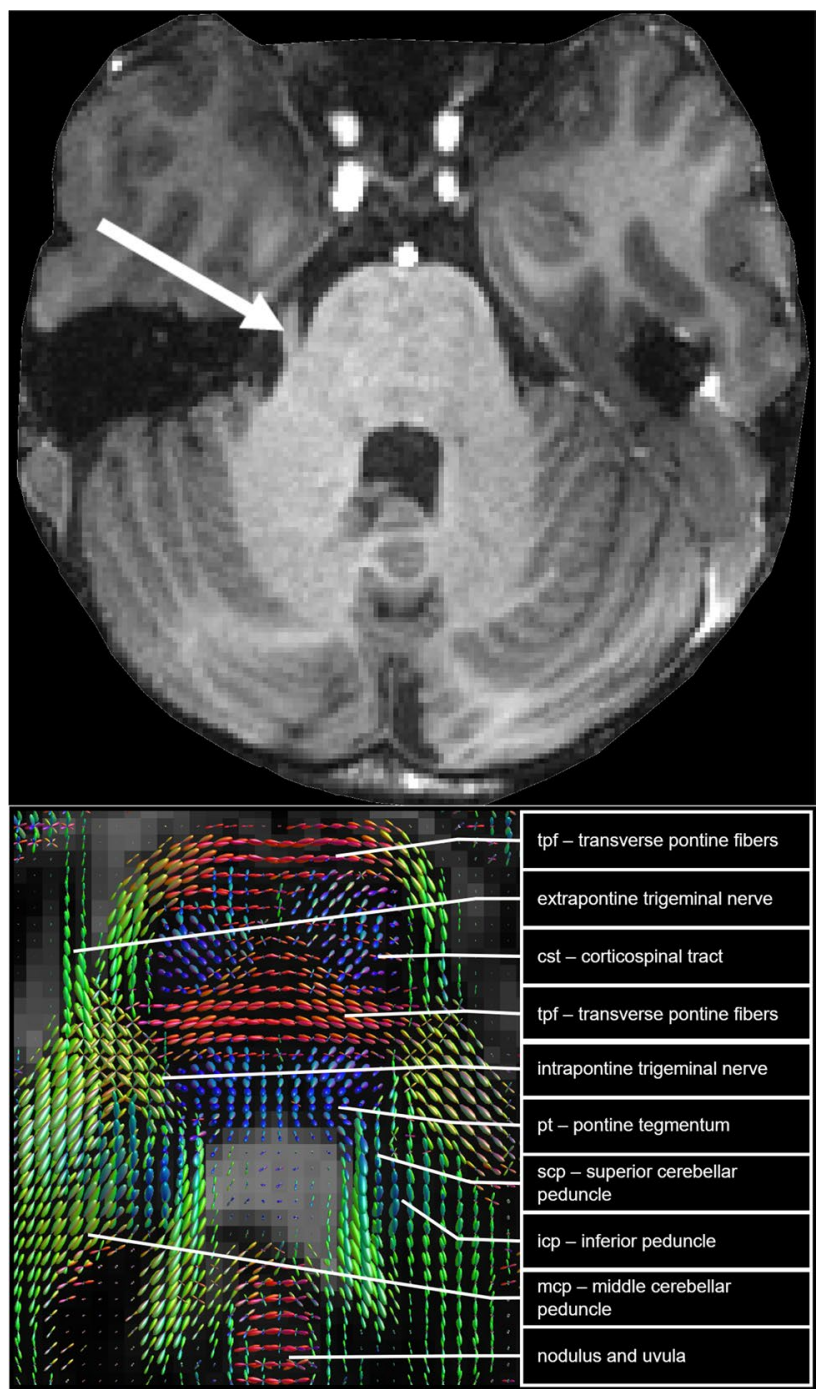

Fig. 2 Pons, trigeminal nerve, and cerebellum (axial orientation, conventions, and color encoding as in Fig. 1). Top: Anatomic image (0.8-mm isotropic resolution) of pontine region with right trigeminal nerve (white arrow points to its extrapontine part). Position corresponds approximately to the Montreal Neurological Institute (MNI) coordinate $z=-27$ ([60], 50-51; [61], 488-489). Bottom: Corresponding color-encoded fiber orientation density functions (fODF) of enlarged pons region showing the right trigeminal nerve leaving or entering, respectively, the pons, overlaid onto the non-diffusionweighted image. The blue fibers [pontine tegmentum $(\mathrm{pt})$ ] include descending and ascending fibers of the central tegmental tract, the medial longitudinal fasciculus, and the medial lemniscus. The large fiber bundles (yellow), which cross the fibers of the middle cerebellar peduncle (mcp) (mainly green) from ventrolateral, represent the intrapontine part of the trigeminal nerve and contain mainly sensory fibers 
anatomic structures. In particular, the fODFs made it possible to distinguish the intrapontine part of the trigeminal nerve when it intersects with surrounding WM parts. The trigeminal nerve itself could be detected in all volunteers (see Fig. S1). Mainly, extra- and intrapontine fibers of the trigeminal nerve were seen in at least two or three adjacent slices, depending on the individual anatomy and applied slice orientation (see Fig. S6). The roots of the left and right trigeminal nerves were for the most part located on different axial planes. Both intra- and extrapontine fibers of the trigeminal nerves could be identified by the direction of their fiber orientation (for details, see caption of Fig. 2).

Figure 3 shows the crossing of the intrapontine parts of the trigeminal nerve in another four volunteers. Despite some minor individual differences, the intrapontine trigeminal nerve fibers and the fibers forming the middle cerebellar peduncles are shown to intersect each other almost orthogonally, thereby forming a dense local network-like pattern.

Figure 4 shows the anatomical localization and corresponding fODF map of the trigeminal intra- and extrapontine fibers. The intrapontine trigeminal nerve fibers can be clearly distinguished from the crossing pontine fibers running into cerebellar peduncles.
The fODF may sometimes be difficult to interpret with respect to the directions of its lobes inside a voxel. However, peak vector amplitude and direction can be extracted and used to calculate angles to the respective coordinate axes or between the main directions of the fODF (peak vectors are directed from the origin of a spherical harmonic to their peak value, thus pointing to the direction of a spherical harmonic lobe). This is demonstrated in Figs. S2 and S3 for the fODF located within the blue rectangle of Fig. 4. Amplitudes of the components represent the relative contribution of the respective fiber populations to the overall diffusion profile within the voxel. All volunteers exhibited crossing dominant lobes of the fODFs where the relative amplitude of the trigeminal part was up to twice as large as the intersecting fiber tracts. The calculated angles between main fiber components in the crossing region showed an almost orthogonal crossing of the intrapontine trigeminal nerve fiber populations with the other local fiber tracts (Figs. S1 and S2). Figure 5 illustrates some results of the fODF analysis for whole-brain data. The fODF maps allow following the orientation characteristics of fibers from cortical GM all the way down to or from subcortical areas, even throughout anatomically complex regions, such as the thalamus, midbrain, and hindbrain, as well as for following the course of fibers to/from the cerebellum.
Fig. 3 Fiber orientation density functions (fODFs) at the intersection between pons, extra- and intrapontine fibers of the trigeminal nerve, and the middle cerebellar peduncles. Shown are representative slices from another four volunteers illustrating the course of intrapontine trigeminal nerve fibers (axial orientation, overlaid on the non-diffusion-weighted image; other conventions and color encoding as in Fig. 1). Intrapontine trigeminal nerve fibers and fibers converging into the middle cerebellar peduncle intersect each other almost orthogonally. For depiction of anatomic structures, see Fig. 2
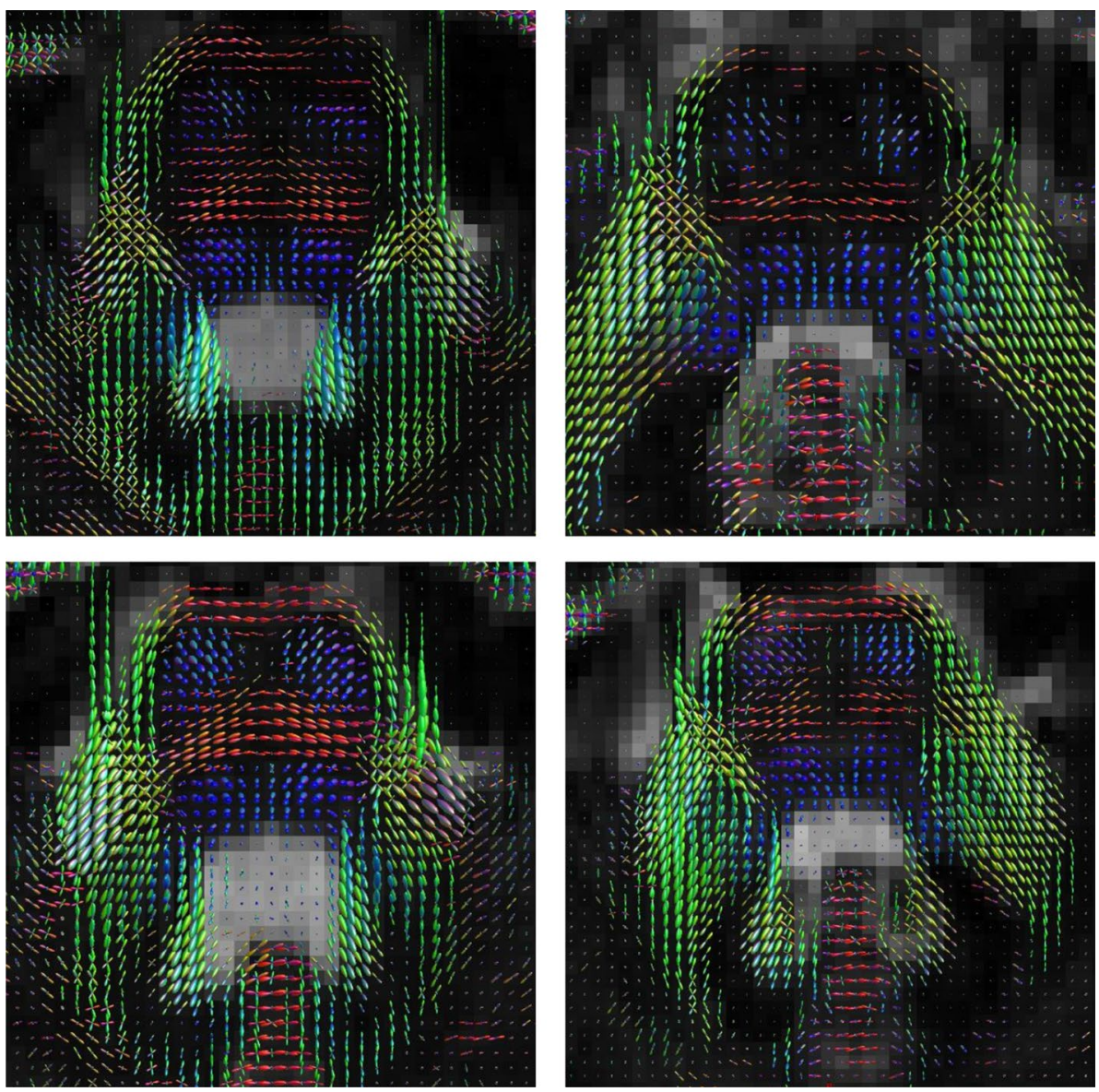
Fig. 4 Details of the voxel-wise fiber orientation distributions within the cerebellopontine region. Top: Sagittal (left) and axial (right) slices of the anatomical magnetization prepared rapid-acquisition gradient echo (MPRAGE) image. The red line in the sagittal view indicates the position of the axial slice. Bottom: Enlarged fiber orientation density functions (fODF) map corresponding to the white rectangle in the axial MPRAGE. The main fiber tracts in the cerebellopontine region are labelled in accordance with Fig. 2. The extrapontine part of the trigeminal nerve (1) along with its intrapontine part (1a, light blue square) can be clearly seen. The intrapontine trigeminal nerve fibers are crossed almost orthogonally (1a) by the transverse pontine fibers (tpf), which continue on to the cerebellar peduncles (2)
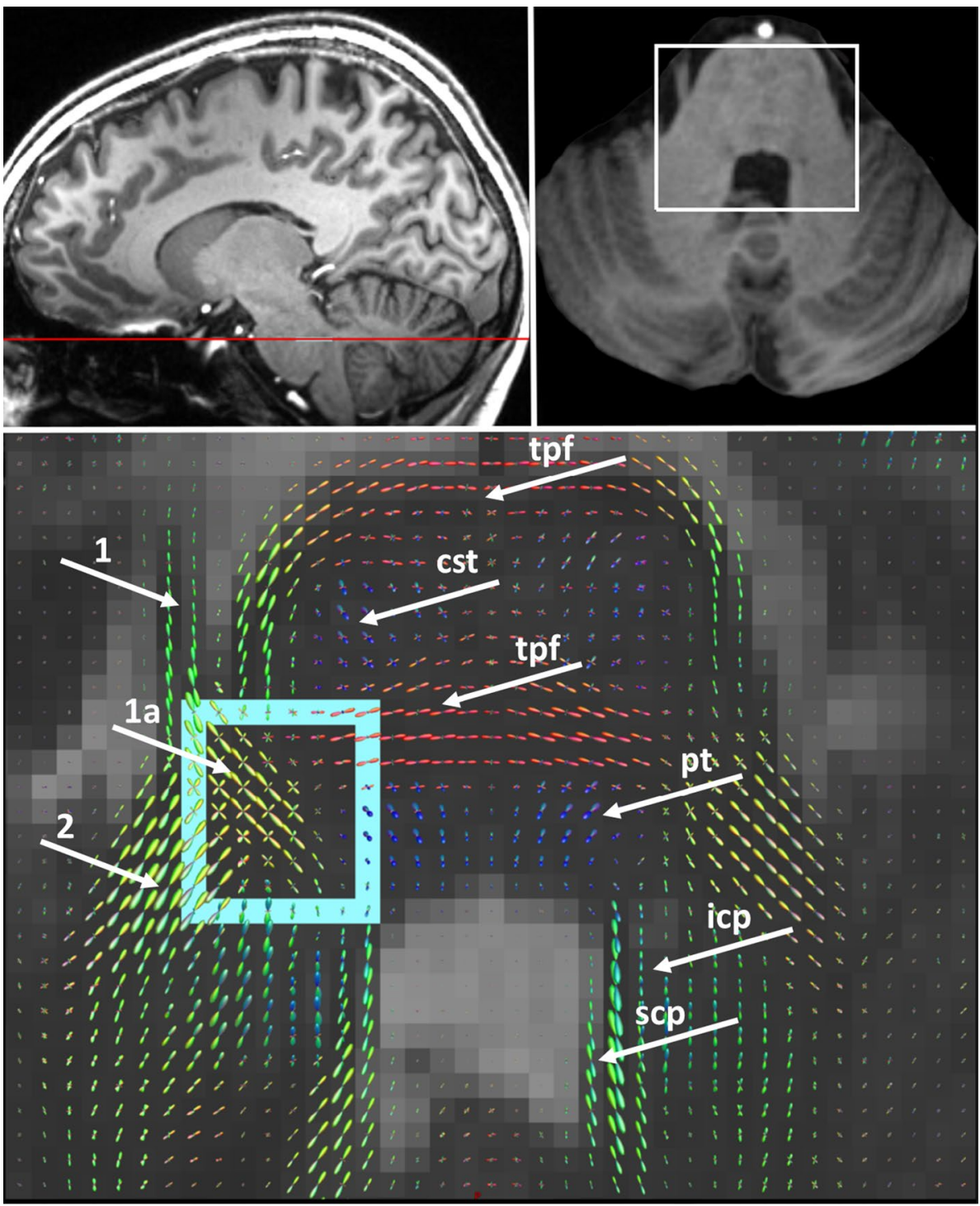

In the cerebellum, fibers connecting the two cerebellar hemispheres (red) and main fiber-forming upper, middle, and lower cerebellar peduncles (green) can be distinguished in the pontine region. The blue-encoded fibers (rostrocaudal direction) represent the crus cerebri (ventral), the medial longitudinal fasciculus, and the central tegmental tract (dorsal), respectively, followed by the spinal cord. The redencoded fibers (left-right direction) between them are the transverse pontine fibers. The superior cerebellar peduncles are seen in the large green-encoded fiber bundle (dorsal-ventral direction) extending from the pons to the cerebellum (in particular, in the sagittal view).

A closer look at the diffusion characteristics in cortical GM revealed region-dependent differences in parts of $\mathrm{S} 1$ and M1. As illustrated in Figs. 6 and 7, we saw a reduced proportion of radially oriented main fODF components in S1 (Brodmann areas 2 and 3) (for orientation: BA1 is located along the crown of the postcentral gyrus, BA2 in the posterior part of the postcentral gyrus that descends into the postcentral sulcus, and BA3 covers the anterior part of the postcentral gyrus that descends into the central sulcus). This supports results of McNab et al. [44], who reported a reduced radially directed anisotropy of the neuronal fibers orthogonal to the cortical surface in S1. In our data, however, the lack of radially oriented fODF components in GM of $\mathrm{S} 1$ appears to be more restricted to the GM of the central part of $\mathrm{S} 1$, which is adjacent to the central sulcus (BA3a/b; Fig. 6, white arrow). The medial and lateral parts of BA3 (orientation with respect to the dorsal view) - and even more so, the posterior part of S1 (BA2, adjacent to the postcentral gyrus; Fig. 6, red arrows)—again suggest a greater proportion of radially oriented main fODF components.

Similarly, in GM of M1 (yellow arrows in Fig. 6) and in the premotor area (green arrows in Fig. 6, including the 

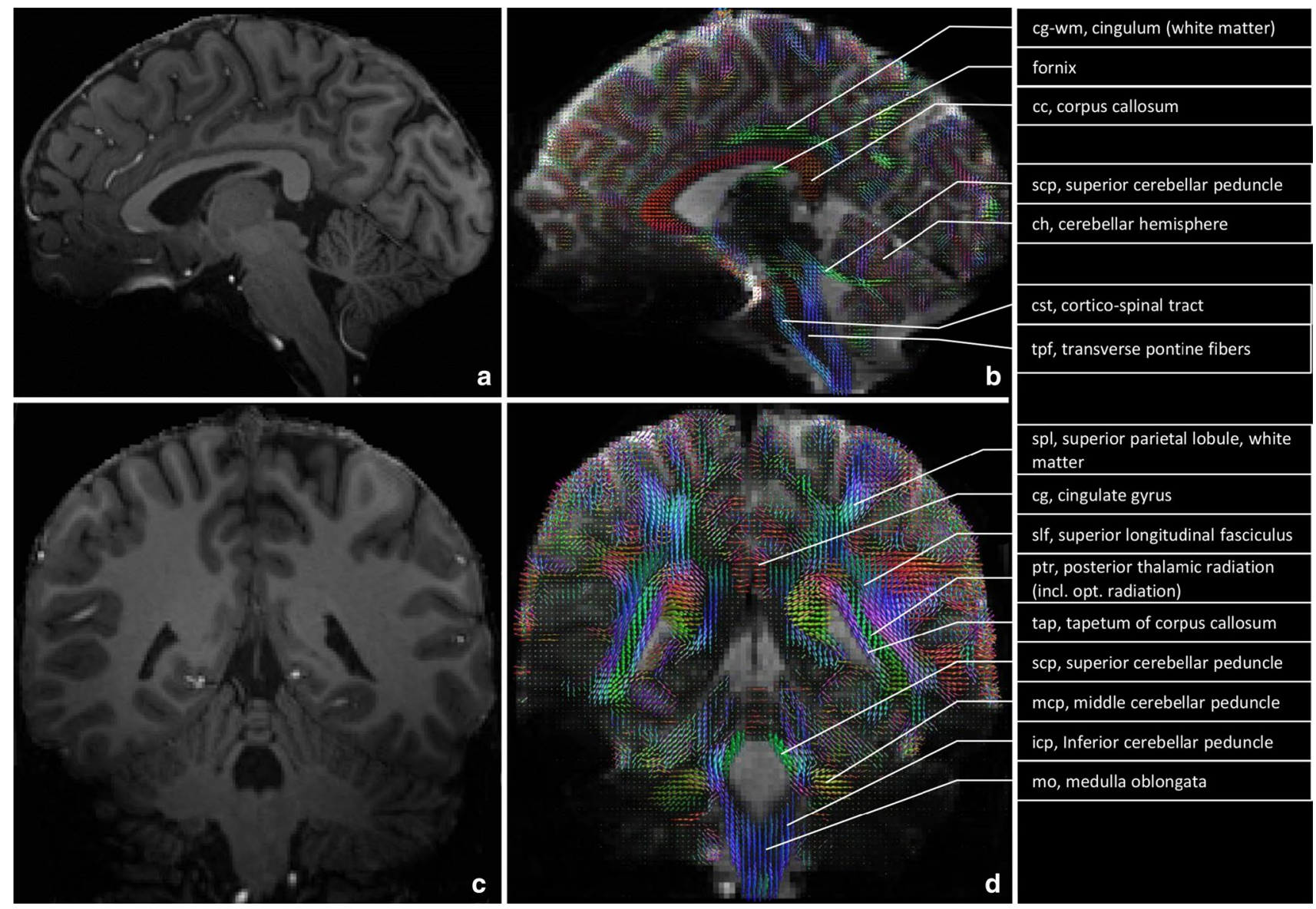

scp, superior cerebellar peduncle

ch, cerebellar hemisphere

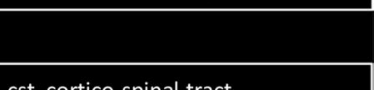

cst, cortico-spinal tract

tpf, transverse pontine fibers

.

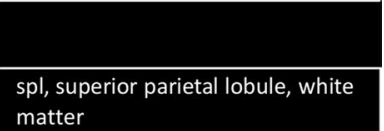

matter

$\mathrm{cg}$, cingulate gyrus

slf, superior longitudinal fasciculus

ptr, posterior thalamic radiation

(incl. opt. radiation)

tap, tapetum of corpus callosum

scp, superior cerebellar peduncle

mcp, middle cerebellar peduncle

icp, Inferior cerebellar peduncle

mo, medulla oblongata

Fig. 5 Example of whole-brain fiber orientation density functions (fODFs). T1-weighted anatomical maps of the whole brain $(0.8-\mathrm{mm}$ isotropic resolution) in sagittal (a) and coronal (c) orientation. Corre-

supplementary motor cortex), extended fiber distributions are found with one dominant radially oriented fODF lobe.

To demonstrate the complex three-dimensional fiber characteristics of the M1/S1 area, fODF maps in three orthogonal views of the M1/S1 region of one representative volunteer are displayed in Fig. 7. In the axial (Fig. 7a) and sagittal (Fig. 7b) slice orientation, we saw no dominant radial fODF component in BA3 of $\mathrm{S} 1$ (white arrow). Again, the fODFs in the central part of S1 suggest dominant fiber orientations that are less perpendicular to the cortical surface, whereas main fiber orientations near the crown of S1 (BA1) and along the postcentral sulcus (BA2) seem to turn perpendicular gain to the cortical surface.

\section{Discussion}

In this study, we demonstrated that a combination of dMRI at 7-T and CSD analysis allows a detailed view of the intravoxel distributions of fiber populations in the whole brain, including cortical and subcortical GM, WM, and GM/WM sponding color-encoded fODF maps $(\mathbf{b}, \mathbf{d})$ overlaid on a T2-weighted image (without diffusion weighting; $1.4 \mathrm{~mm}$ isotropic resolution). For color encoding, see Fig. 1)

transition zones. In particular, intrapontine fibers of the trigeminal nerve could be distinguished from crossing fibers of the surrounding cerebellopontine tracts. We also provided evidence that in cortical GM, the orientation of fODFs of $\mathrm{S} 1$ seems to be less perpendicular to the cortical surface than in GM of M1, premotor, and secondary somatosensory cortices. This is discussed now in more detail.

\section{Trigeminal nerve}

We took advantage of the increased SNR of dMRI at 7-T compared with 3-T MRI and of the potential of fODFs to resolve fine-scale structures in the brain, such as in the cerebellopontine angle. Pathological changes to the extrapontine parts of the trigeminal nerve were reported [38, 39, 41], and the necessity of fiber-tracking its intra- and extrapontine parts was recently reported while comparing different algorithms, including CSD [42].

Analysis of the fODF makes it possible to estimate the partial contributions and exact orientation of corresponding fiber populations (Fig. S2) in each voxel. The 
Fig. 6 Spatial distribution of fiber orientation density functions (fODF) in gray matter (GM) of the premotor, motor, and somatosensory cortices. Color-encoded fODF map overlaid on a T2-weighted image (without diffusion weighting, 1.4-mm isotropic resolution). With the exception of in the central parts of the primary somatosensory cortex (S1, $\mathrm{BA} 3 \mathrm{a} / \mathrm{b}$, white arrow), fODF distributions in parts of the cortical GM suggest an orientation predominantly perpendicular to the cortical surface (called radial fiber orientation). In addition to the GM of the primary motor cortex (M1; yellow arrows), the GM of the premotor region (BA6, BA8; anterior to $\mathrm{M} 1$, green arrows), the posterior part of S1 (BA2; adjacent to the postcentral gyrus, red arrows), and the secondary somatosensory cortices (BA5, BA7; posterior to S1) exhibit a mostly radial orientation of the dominant fODF lobe
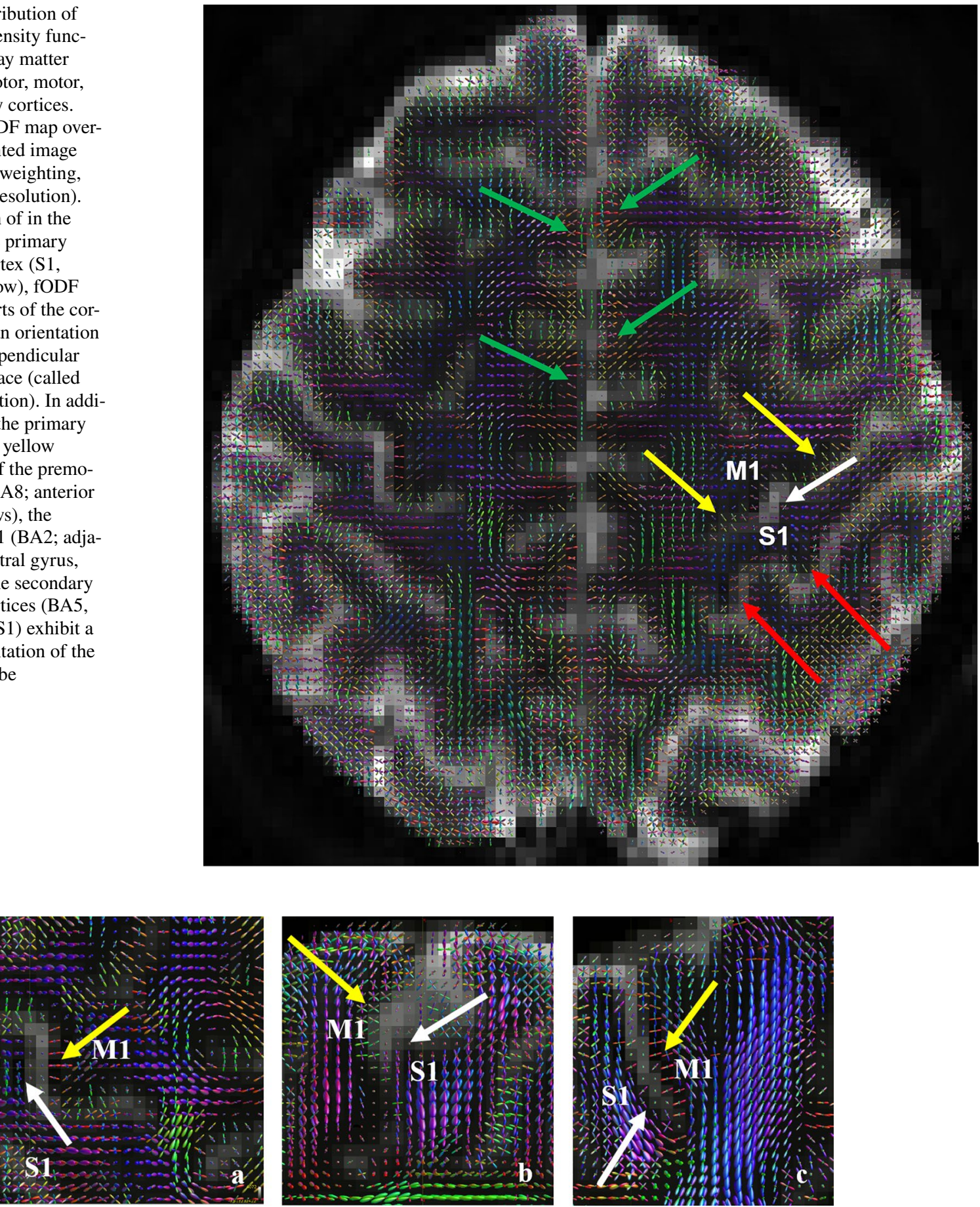

Fig. 7 Three orthogonal views of the right primary motor (M1) and somatosensory regions (S1). Axial (a), sagittal (b), and coronal (c) views of color-encoded fiber orientation density functions (fODF) maps overlaid onto the corresponding non-diffusion-weighted image data (1.4-mm isotropic resolution). White-matter (WM) tracts in the

fODF attributed here to the extrapontine trigeminal fibers exhibited only one dominant lobe that could be followed continuously when traversing the pons to the dorsal pons that was attributed to representing the intrapontine fibers depicted parts of M1 and S1 are predominantly oriented in rostrocaudal direction (blue to violet). The fODF in the central part of S1 (BA3) suggests fewer radially oriented main fODF components (a, b, white arrows) than in M1 (yellow arrows)

of the trigeminus. These intrapontine voxels exhibited a second, nearly orthogonally directed, fODF lobe, which was interpreted as belonging to the transverse pontine fibers crossing the intrapontine trigeminal fibers and 
turning into the middle cerebral peduncle. Thus, we show here using noninvasive in vivo imaging how intrapontine trigeminal nerve fibers cross with other fiber populations connecting the pons with the cerebellar peduncles. In particular, the course of intrapontine fibers seen in our images fits well with the anatomy of the thick sensory fibers from the Radix sensoria, conveying epicritic and proprioceptive information to the principal and mesencephalic trigeminal nuclei located dorsal and only slightly caudal to the point where the trigeminal nerve enters/ leaves the pons. These intrapontine fibers might also comprise the thick motor fibers from the neighboring trigeminal motor nucleus, which initially traverse together with fibers from the mesencephalic trigeminal nucleus as tractus mesencephalicus and later form the Radix motoria. We could not unequivocally distinguish the thin sensory fibers of the tractus spinalis nervi trigemini (protopathic sensibility) that bend toward the more caudally located spinal trigeminal nucleus.

The diffusion properties of the trigeminal nerve and other CNs can serve to help detect neuronal pathologies due to virus-induced inflammation or radiotherapy [35-38, 40, 41]. Future studies will show whether this is also applicable to other pathologic processes, such as multiple sclerosis. The techniques described in our study may even add more information concerning fine-scale lesions following trauma, thereby making it possible to distinguish between external and internal $\mathrm{CN}$ lesions and fiber lesions of local brainstem tissue.

Spatial high-resolution dMRI can also serve to track the course of CNs: Behan et al. [42] reported 3-T MRI data of ten patients with posterior fossa tumors where the CNs $\mathrm{V}$ (trigeminus) and VII/VIII (facial/vestibular complex) were tracked with a resolution of $0.94 \times 0.94 \times 3.0 \mathrm{~mm}^{3}$ and diffusion weighting of $b=1000 \mathrm{~s} / \mathrm{mm}^{2}$. They compared three tracking algorithms (one based on CSD) focusing on results of fiber tracking algorithms. Orientation characteristics of intrapontine fODFs were not further discussed. However, our data, with lower in-plane $\left(1.4 \mathrm{~mm}^{2}\right)$ but higher through-plane resolution of $1.4 \mathrm{~mm}$ and good SNR at $7 \mathrm{~T}$, enabled intrapontine trigeminal fODF characteristics to be detected in typically two to four slices in all but one volunteer. Due to this high resolution, intrapontine trigeminal fiber populations interestingly show an intertwined pattern with the surrounding WM tracts instead of forming a homogeneous bundle with only one dominant fODF component. This suggests that the intrapontine trigeminal fiber populations are interwoven with the surrounding pontine WM tracts instead of forming a solid separable fiber bundle in the pons. This fits with the well-known fact that the origin and target of trigeminus fibers lie in several pontine nuclei.

\section{Cortical gray matter}

Our study supports previous findings about local differences in diffusion anisotropy in cortical GM, such as in McNab et al. [44], who showed that in many cortical areas, fibers are directed mainly perpendicular (radial) to the complex, three-dimensionally folded cortical surface [18, 45, 47, 51, $62,63]$. Our finding that radially oriented fODF components are lacking in the central part of S1 (BA 3a/b) but are seen in primary somatosensory areas BA 1 and BA 2 is reproducible in the orthogonal slice positions (Fig. 7). This suggests that radially oriented fODF components are not an artefact due to projection of the fODF onto one plane. Nevertheless, it may not completely be ruled out that other factors, such as noise, may influence fODF analysis.

Pronounced radiallydirected fODF components can also be found throughout the GM of the secondary somatosensory cortex (BA5, BA7) as well as in the GM of M1 and the premotor cortex (BA6, BA8; Fig. 6). The varying diffusion characteristics of cortical GM most likely reflect different fiber and cytoarchitectural features of these areas. In the cortical GM, neuronal cells are embedded in a dense matrix of glial cells and vessels that build a layered structure composed of several cell types. Although the neurites run mainly perpendicular to the folded cortical surface, there are dense horizontal connections between cortical neurons (as in S1 [64]).

In the ex vivo study of Leuze et al. [50], micro-MRI data of a human specimen of visual cortex were acquired with an isotropic resolution of $0.242 \mathrm{~mm}$. Subsequently, the derived diffusion information (including fODF) was compared with corresponding histological images. The authors succeeded in resolving the band-like tangential diffusion anisotropy of the stripe of Gennari in V1. In addition, they reported tangential structures near the cortical surface that most likely correspond to cortical long-range association fibers. Although the GM of human in vivo M1 and S1 are $~ 3.9$ and $1.8-\mathrm{mm}$ thick, respectively [49], the cortical GM is sometimes projected obliquely onto the slices, causing an apparently larger extension of the GM (see premotor cortical areas in Fig. 5). Interestingly, the absence of radially directed fODF peak vectors in our study is most pronounced in the central part of S1 (BA3a/b), but the fraction of radially oriented fODF peak vectors increases again in its other parts and in BA1 and BA2 (Figs. 6, 7).

Calamante et al. [51] recently used multishell, multitissue (MSMT) CSD of 1.25-mm isotropic resolution data acquired at $3 \mathrm{~T}$ with increasing diffusion weighting $(b=1000,2000$, $3000 \mathrm{~s} / \mathrm{mm}^{2}$ ) to estimate apparent fiber densities (AFD) in a group of eight healthy individuals. The results of the MSMTCSD-based ODF analysis were used to describe different fiber architectures of cortical GM, particularly in M1 and S1. Similar to our findings, GM in M1 was found to exhibit 
a primarily radially dominant fiber orientation, whereas GM in S1 mostly shows a tangential orientation. Kleinnijenhuis et al. [63] acquired 1-mm isotropic data at $7 \mathrm{~T}$ with sagittal orientation in a slab centered on the interhemispheric fissure and with a diffusion weighting of $b=1000 \mathrm{~s} / \mathrm{mm}^{2}$. The authors calculated a radiality measure after defining several layers in the cortical GM and the adjacent WM. They argue that radiality depends both on the position of the voxel with respect to the cortical layer and on the location of the voxel between crown, banks, and fundus of the gyrus. Radiality was largest in intermediate cortical layers located next to the crown, but not on the crown or the WM itself. Tangential tensor orientation was prominent in the deep cortical layers in the fundus. In WM, diffusion tensor orientation was radial on the crown and changed to tangential under the banks and fundus. These findings also support our results (Fig. 7).

As stated above, the different features of the fODFs in M1 and S1 most likely reflect their microarchitectural differences. M1 contains only five clearly separable cell layers; it also contains pyramidal cells ranging from small to giant (Beetz cells). There is no clear layer IV composed of granular cells. The axons of the M1 pyramidal cells are already myelinated and converge via the corona radiata and cerebral peduncles mainly into the pyramidal tract. S1 has six distinguishable layers and thus contains many granular cells (layer IV). Tangentially oriented structures seen in layers II and IV of S1 consist of horizontal nerve fibers (band of Kaes-Bechterew, outer band of Baillarger, respectively). Tangential structures are also present on the cortical surface (Exner's stripe) and represent the long-range corticocortical connections between the supragranular layers.

The fODF maps show in detail the fine-scale fiber population orientation within the GM and across the GM/WM borders: between gyral crowns and the WM, fibers usually are straight, while the dominant fODF orientation changes at the gyral banks before forming WM tracts. With regard to the potential of high-resolution dMRI combined with fODF and the corresponding visualization of results, this represents a major step toward in vivo, high-resolution mapping of microstructural brain tissue architectures [55, 57, 65].

\section{Limitations and possible solutions}

There are some limitations to our study. We examined a rather homogeneous group of young, healthy volunteers; including elderly people may alter some of the results. Next, despite convincing results, the analysis of fiber population directions based on anisotropic diffusion characteristics in non-WM tissue, such as the GM-which contains more diverse components (e.g., dendrites, vessels) and are thus microarchitecturally much more complex-should be approached with some caution. Thus, depending on the tissue, the term "anisotropic diffusion" may, instead, refer to varying anatomic substructures rather than to neuronal fibers only.

The applied protocol also has technical limitations. It is well known that echo-planar imaging at ultrahigh frequency (UHF) exhibits some measurement-related problems, most significantly, geometric distortions. Strong local variations in susceptibility may play a role in the frontal region (especially if an individual has large, frontal air-filled sinuses) in the lower temporal regions and at the brain base. We did correct for geometric distortion by acquiring and using one additional non-diffusion-weighted field map but refrained from using more sophisticated correction techniques, such as applying point-spread function correction [66] or acquiring additional data sets with reversed phase-encoded directions [23, 24], because these considerably prolong acquisition and reconstruction times. The chosen resolution was also a compromise between measurement time, whole-head coverage, and image quality [52]. However, we showed in a previous study that if fewer slices in reduced volumes of interest are acquired or if clinical findings allow for limiting of data acquisition to reduce volumes of interest, then higher-resolution techniques such as zoomed imaging and partially parallel acquisition (ZOOPPA) can be applied successfully $[15,17]$.

Multiband techniques, adapted pulses, and optimized acquisition schemes can reduce acquisition time even further. In the human connectome project, data acquisition in healthy volunteers was optimized for anatomic and diffusion MRI [30-32]. This made it possible to obtain 1.05-mm isotropic whole-head 7-T dMRI data in $~ 40$ min when acquiring two different phase-encoding data sets to compensate for geometrical distortions [23]. One of the major steps toward faster data acquisition is the use of multiband dMRI [17, 22, 30, 31, 67]. There is also some debate whether 3-T MR scanners with higher gradient strengths are superior to 7-T MR scanners, given that the latter have some drawbacks, especially shortened $\mathrm{T} 2 \times$ time and higher deposition of radiofrequency (RF) energy into the tissue [specific absorption rate (SAR)]. As a consequence, quality of diffusion data was further improved within the human connectome project by combining the advantages of both 3-T and 7-T diffusion imaging.

Although we used only one diffusion-weighted data set for data processing, CSD can profit from including multiple and higher diffusion-weighted data conditions [51]. However, calculation of the apparent diffusion constant (ADC), which we used to differentiate CSF from GM voxels (Fig. $\mathrm{S} 5$ ), is less reliable at high $b$ values, due to the increased influence of noise.

For postprocessing, new algorithms [25] and the use of additional tissue-characterizing data, such as susceptibilityweighted images to detect, for example, pontine nuclei [68, 69], may improve the identification of fine-scale anatomic 
structures of CNs. However, the advantages of acquiring and postprocessing multishell data $[56,70]$ must be balanced against potential drawbacks. When examining patients, prolonged acquisition time may increase the probability of patient movements that, in turn, can diminish data quality, given that diffusion imaging is highly motion sensitive. Other constraints include limitations due to SAR as well as a small decrease in SNR for multiband techniques [30]. A thorough discussion of the different effects for 7-T diffusion imaging and the different strategies for optimization is provided by $\mathrm{Vu}$ et al. and Sotiropulus et al. [23, 30]. An interesting alternative strategy to increase resolution while keeping acquisition time short was demonstrated by Dyrby et al. [71], who interpolated ex vivo dMRI data of monkey brain and clinical human dMRI data prior to fiber reconstruction. Except for tract boundaries or when strong partial volume effects were present, more details could be seen; this was validated by additional simulations.

Although the measurement time of $\sim 50 \mathrm{~min}$ for the whole head may be debatable in a clinical setting, our findings may serve as a starting point for further optimization with respect to data quality and gain of diagnostic information. Data acquisition of reduced volumes of interest, as in our study, or applying simultaneous multiband acquisition techniques, will considerably shorten the acquisition time $[15,17,22$, 24, 30, 67].

Finally, the fODF can distinguish differently oriented fiber populations within one voxel under the condition that the angle is larger than $\sim 40^{\circ}$ [27]. Also, partial volume effects, such as at the border between GM and cerebrospinal fluid (CSF), play a role. For example, voxels with isotropic distribution of the fODF components were differentiated as belonging to CSF by assuming their T2-weighted values or $\mathrm{ADC}$ values to be similar to those in Bernarding et al. [4]. CSF exhibits clearly elevated ADC values, although - due to partial volume effects with GM - they rarely reach values comparable with $\sim 3000 \mathrm{~mm}^{2} / \mathrm{s}$, as within larger CSF spaces (Fig. S5). Thus, T2-weighted or ADC values may serve as complementary information about whether voxels belong mainly to CSF, GM, or WM or whether they represent a mixture of different tissue classes.

\section{Conclusion}

We demonstrated the feasibility of whole-brain CSD applied to UHF dMRI with 1.4-mm isotropic resolution; fODF could be determined, allowing analysis of orientations of fiber populations within cortical GM, WM, subcortical areas such as the cerebellopontine angle, and the cerebellum. In all but one volunteer, the intrapontine parts of the trigeminal nerve were identified by the direction of the main fODF lobe intersecting near-orthogonally with the main fODF lobes of the surrounding brainstem fiber tracts. Additionally, we found evidence that in parts of the primary somatosensory cortex the main component of each fODF seems to be oriented less perpendicular to the cortical surface than in GM of remaining motor, premotor, and secondary somatosensory cortices.

With 7-T MRI currently being introduced into clinical routine, high-resolution diffusion may not only characterize fine-scale anatomic structures but also provide additional evidence in small-scale pathologies of GM and WM due to traumatic, neurodegenerative, or inflammatory processes.

Author contributions Study conception and design: Bernarding, Luetzkendorf, Data acquisition: Luetzkendorf, Kaufmann, Baecke, Stadler, Heidemann, Feiweier. Dt analysis and interpretation: Luetzkendorf, Bernarding, Budinger, Luchtmann. Manuscript drafting: Luetzkendorf, Bernarding, Heidemann, Luchtmann. MR sequence development: Feiweier, Heidemann. Critical revision: Luetzkendorf, Bernarding, Heidemann, Feiweier, Luchtmann, Stadler, Kaufmann, Baecke, Budinger, A data set with 1.4-mm isotropic resolution can be obtained upon request.

\section{Compliance with ethical standards}

Conflict of interest Heidemann and Feiweier are employees of Siemens Healthcare GmbH. Both hold Siemens shares and have issued Siemens patents. The other authors declare no competing financial interests.

Ethical standards All procedures performed in studies involving human participants were in accordance with the ethical standards of the institutional and/or national research committee and with the 1964 Helsinki declaration and its later amendments or comparable ethical standards.

Open Access This article is distributed under the terms of the Creative Commons Attribution 4.0 International License (http://creativeco mmons.org/licenses/by/4.0/), which permits unrestricted use, distribution, and reproduction in any medium, provided you give appropriate credit to the original author(s) and the source, provide a link to the Creative Commons license, and indicate if changes were made.

\section{References}

1. Le Bihan D, Breton E, Lallemand D et al (1986) MR imaging of intravoxel incoherent motions: application to diffusion and perfusion in neurologic disorders. Radiology 161(2):401-407. https:// doi.org/10.1148/radiology.161.2.3763909

2. Stejskal EO, Tanner JE (1965) Spin diffusion measurements: spin echoes in the presence of a time-dependent field gradient. J Chem Phy 42(1):288. https://doi.org/10.1063/1.1695690

3. Moseley ME, Kucharczyk J, Mintorovitch J et al (1990) Diffusion-weighted MR imaging of acute stroke: correlation with T2-weighted and magnetic susceptibility-enhanced MR imaging in cats. AJNR Am J Neuroradiol 11(3):423-429

4. Bernarding J, Braun J, Hohmann J et al (2000) Histogram-based characterization of healthy and ischemic brain tissues using multiparametric MR imaging including apparent diffusion coefficient maps and relaxometry. Magn Reson Med 43(1):52-61

5. Koennecke HC, Bernarding J (2001) Diffusion-weighted magnetic resonance imaging in two patients with polycythemia rubra vera and early ischemic stroke. Eur J Neurol 8(3):273-277 
6. Moseley ME, Cohen Y, Kucharczyk J et al (1990) Diffusionweighted MR imaging of anisotropic water diffusion in cat central nervous system. Radiology 176(2):439-445. https://doi. org/10.1148/radiology.176.2.2367658

7. Siewert B, Patel MR, Warach S (1995) Stroke and ischemia. Magn Reson Imaging Clin N Am 3(3):529-540

8. Warach S, Dashe JF, Edelman RR (1996) Clinical outcome in ischemic stroke predicted by early diffusion-weighted and perfusion magnetic resonance imaging: a preliminary analysis. J Cereb Blood Flow Metab 16(1):53-59. https://doi.org/10.1097/00004 647-199601000-00006

9. Assaf Y, Freidlin RZ, Rohde GK et al (2004) New modeling and experimental framework to characterize hindered and restricted water diffusion in brain white matter. Magn Reson Med 52(5):965-978. https://doi.org/10.1002/mrm.20274

10. Basser PJ, Pajevic S, Pierpaoli $C$ et al (2000) In vivo fiber tractography using DT-MRI data. Magn Reson Med 44(4):625-632

11. Mori S, Crain BJ, Chacko VP et al (1999) Three-dimensional tracking of axonal projections in the brain by magnetic resonance imaging. Ann Neurol 45(2):265-269

12. Pierpaoli C, Basser PJ (1996) Toward a quantitative assessment of diffusion anisotropy. Magn Reson Med 36(6):893-906

13. Virta A, Barnett A, Pierpaoli C (1999) Visualizing and characterizing white matter fiber structure and architecture in the human pyramidal tract using diffusion tensor MRI. Magn Reson Imaging 17(8):1121-1133

14. van Wedeen J, Rosene DL, Wang R et al (2012) The geometric structure of the brain fiber pathways. Science 335(6076):1628 1634. https://doi.org/10.1126/science. 1215280

15. Heidemann RM, Ivanov $D$, Trampel $R$ et al (2012) Isotropic submillimeter fMRI in the human brain at $7 \mathrm{~T}$ : combining reduced field-of-view imaging and partially parallel acquisitions. Magn Reson Med 68(5):1506-1516. https://doi.org/10.1002/mrm.24156

16. Luetzkendorf R, Heidemann R, Feiweier T et al (2016) Spherical deconvolution of high-resolution $7 \mathrm{~T}$ whole-head diffusion magnetic resonance images shows reduced radial anisotropic diffusion in human primary somatosensory cortex. In: Annual Meeting of the International Society of Magnetic Resonance in Medicine, 2016

17. Eichner C, Setsompop K, Koopmans PJ et al (2014) Slice accelerated diffusion-weighted imaging at ultra-high-field strength. Magn Reson Med 71(4):1518-1525. https://doi.org/10.1002/mrm.24809

18. Heidemann RM, Anwander A, Eichner C et al (2011) Isotropic sub-millimeter diffusion MRI in humans at $7 \mathrm{~T}$. In: Proceedings of the organisation of human brain mapping, Québec City, Canada, 26-30 June 2011

19. Heidemann RM, Anwander A, Feiweier T et al (2012) k-space and q-space: combining ultra-high spatial and angular resolution in diffusion imaging using ZOOPPA at $7 \mathrm{~T}$. Neuroimage 60(2):967978. https://doi.org/10.1016/j.neuroimage.2011.12.081

20. Prčkovska V, Achterberg HC, Bastiani M et al (2013) Optimal short-time acquisition schemes in high angular resolution diffusion-weighted imaging. Int J Biomed Imaging 2013:658583. https ://doi.org/10.1155/2013/658583

21. Tournier J-D, Calamante F, Connelly A (2012) MRtrix: diffusion tractography in crossing fiber regions. Int J Imaging Syst Technol 22(1):53-66. https://doi.org/10.1002/ima.22005

22. Setsompop K, Kimmlingen R, Eberlein E et al (2013) Pushing the limits of in vivo diffusion MRI for the human connectome project. Neuroimage 80:220-233. https://doi.org/10.1016/j.neuro image.2013.05.078

23. Vu AT, Auerbach E, Lenglet C et al (2015) High resolution whole brain diffusion imaging at $7 \mathrm{~T}$ for the human connectome project. Neuroimage 122:318-331. https://doi.org/10.1016/j.neuroimage .2015 .08 .004
24. Sotiropoulos SN, Hernández-Fernández M, Vu AT et al (2016) Fusion in diffusion MRI for improved fibre orientation estimation: an application to the $3 \mathrm{~T}$ and $7 \mathrm{~T}$ data of the human connectome project. Neuroimage 134:396-409. https://doi.org/10.1016/j.neuro image.2016.04.014

25. Dell'Acqua F, Scifo P, Rizzo G et al (2010) A modified damped Richardson-Lucy algorithm to reduce isotropic background effects in spherical deconvolution. Neuroimage 49(2):1446-1458. https://doi.org/10.1016/j.neuroimage.2009.09.033

26. Tournier J-D, Calamante F, Gadian DG et al (2004) Direct estimation of the fiber orientation density function from diffusionweighted MRI data using spherical deconvolution. Neuroimage 23(3):1176-1185. https://doi.org/10.1016/j.neuroimage .2004 .07 .037

27. Tournier J-D, Calamante F, Connelly A (2007) Robust determination of the fibre orientation distribution in diffusion MRI: nonnegativity constrained super-resolved spherical deconvolution. Neuroimage 35(4):1459-1472. https://doi.org/10.1016/j.neuro image.2007.02.016

28. Ros C, Güllmar D, Stenzel M et al (2013) Atlas-guided cluster analysis of large tractography datasets. PLoS One 8(12):e83847. https://doi.org/10.1371/journal.pone.0083847

29. Assaf Y, Alexander DC, Jones DK et al (2013) The CONNECT project: combining macro- and micro-structure. Neuroimage 80:273-282. https://doi.org/10.1016/j.neuroimage.2013.05.055

30. Sotiropoulos SN, Jbabdi S, Xu J et al (2013) Advances in diffusion MRI acquisition and processing in the Human Connectome Project. Neuroimage 80:125-143. https://doi.org/10.1016/j.neuro image.2013.05.057

31. Uğurbil K, Xu J, Auerbach EJ et al (2013) Pushing spatial and temporal resolution for functional and diffusion MRI in the human connectome project. Neuroimage 80:80-104. https://doi. org/10.1016/j.neuroimage.2013.05.012

32. van Essen DC, Smith SM, Barch DM et al (2013) The WU-Minn human connectome project: an overview. Neuroimage 80:62-79. https://doi.org/10.1016/j.neuroimage.2013.05.041

33. Glasser MF, Smith SM, Marcus DS et al (2016) The human connectome project's neuroimaging approach. Nat Neurosci 19(9):1175-1187. https://doi.org/10.1038/nn.4361

34. Jbabdi S, Sotiropoulos SN, Haber SN et al (2015) Measuring macroscopic brain connections in vivo. Nat Neurosci 18(11):15461555. https://doi.org/10.1038/nn.4134

35. Hodaie M, Chen DQ, Quan J et al (2012) Tractography delineates microstructural changes in the trigeminal nerve after focal radiosurgery for trigeminal neuralgia. PLoS One 7(3):e32745. https:// doi.org/10.1371/journal.pone.0032745

36. DeSouza DD, Hodaie M, Davis KD (2014) Diffusion imaging in trigeminal neuralgia reveals abnormal trigeminal nerve and brain white matter. Pain 155(9):1905-1906. https://doi.org/10.1016/j. pain.2014.05.026

37. DeSouza DD, Hodaie M, Davis KD (2016) Structural magnetic resonance imaging can identify trigeminal system abnormalities in classical trigeminal neuralgia. Front Neuroanat 10:95. https:// doi.org/10.3389/fnana.2016.00095

38. Hodaie M, Quan J, Chen DQ (2010) In vivo visualization of cranial nerve pathways in humans using diffusion-based tractography. Neurosurgery 66(4):788-795. https://doi.org/10.1227/01. neu.0000367613.09324.da (discussion 795-796)

39. Erbay SH, Bhadelia RA, O'Callaghan M et al (2006) Nerve atrophy in severe trigeminal neuralgia: noninvasive confirmation at MR imaging-initial experience. Radiology 238(2):689-692. https ://doi.org/10.1148/radiol.2382042214

40. Krishna V, Sammartino F, Yee P et al (2016) Diffusion tensor imaging assessment of microstructural brainstem integrity in Chiari malformation Type I. J Neurosurg 125(5):1112-1119. https:// doi.org/10.3171/2015.9.jns 151196 
41. Rousseau A, Nasser G, Chiquet C et al (2015) Diffusion tensor magnetic resonance imaging of trigeminal nerves in relapsing herpetic keratouveitis. PLoS One 10(4):e0122186. https://doi. org/10.1371/journal.pone.0122186

42. Behan B, Chen DQ, Sammartino F et al (2017) Comparison of diffusion-weighted MRI reconstruction methods for visualization of cranial nerves in posterior fossa surgery. Front Neurosci 11:554. https://doi.org/10.3389/fnins.2017.00554

43. Walker L, Chang L-C, Nayak A et al (2015) The diffusion tensor imaging (DTI) component of the NIH MRI study of normal brain development (PedsDTI). Neuroimage. https://doi.org/10.1016/j. neuroimage.2015.05.083

44. McNab JA, Polimeni JR, Wang R et al (2013) Surface based analysis of diffusion orientation for identifying architectonic domains in the in vivo human cortex. Neuroimage 69:87-100. https://doi. org/10.1016/j.neuroimage.2012.11.065

45. Anwander A, Pampel A, Knösche TR (2010) In vivo measurement of cortical anisotropy by diffusion-weighted imaging correlates with cortex type. In: ISMRM Joint Annual Meeting. Stockholm, Sweden, 1-7 May 2010, p 109

46. Calamante F, Oh S-H, Tournier J-D et al (2013) Super-resolution track-density imaging of thalamic substructures: comparison with high-resolution anatomical magnetic resonance imaging at 7.0T. Hum Brain Mapp 34(10):2538-2548. https://doi.org/10.1002/ hbm. 22083

47. Luetzkendorf R, Hertel F, Heidemann R et al (2013) noninvasive high-resolution tracking of human neuronal pathways: diffusion tensor imaging at $7 \mathrm{~T}$ with $1.2 \mathrm{~mm}$ isotropic voxel size: medical imaging 2013: physics of medical imaging, edited by Robert M. Nishikawa, Bruce R. Whiting, Christoph Hoeschen. Proc SPIE 8668:866846. https://doi.org/10.1117/12.2006764

48. Truong T-K, Guidon A, Song AW (2014) Cortical depth dependence of the diffusion anisotropy in the human cortical gray matter in vivo. PLoS One 9(3):e91424. https://doi.org/10.1371/journ al.pone.0091424

49. Zilles K, Amunts K (2012) Architecture of the Cerebral Cortex. Human Nerv Syst. https://doi.org/10.1016/B978-0-12-37423 6-0.10023-9

50. Leuze CWU, Anwander A, Bazin P-L et al (2014) Layer-specific intracortical connectivity revealed with diffusion MRI. Cereb Cortex 24(2):328-339. https://doi.org/10.1093/cercor/bhs311

51. Calamante F, Jeurissen B, Smith RE et al (2018) The role of whole-brain diffusion MRI as a tool for studying human in vivo cortical segregation based on a measure of neurite density. Magn Reson Med 79(5):2738-2744. https://doi.org/10.1002/mrm.26917

52. Luetzkendorf R, Heidemann R, Anwander A et al (2012) DWI at $7 \mathrm{~T}$ with a high performance gradient system and a 32 channel head coil: resolution vs time. In: 18th Annual Meeting of the International Society of Magnetic Resonance in Medicine

53. Luetzkendorf R, Heidemann R, Feiweier T et al (2015) Analysis of gray matter anisotropy using spherical deconvolution of $7 \mathrm{~T}$ diffusion-weighted MR images. In: 21st Annual Meeting of the Organization of Human Brain Mapping, Honolulu, USA, 2015

54. Luetzkendorf R, Heidemann R, Feiweier T et al (2015) Analysis of neuronal fiber orientation distribution in gray matter and at gray-white matter borders using spherical deconvolution of highresolution $\left(1.4 \mathrm{~mm}^{3}\right) 7 \mathrm{~T}$ DWI data. In: Annual Meeting of the International Society of Magnetic Resonance in Medicine, 2015
55. Morelli JN, Runge VM, Feiweier T et al (2010) Evaluation of a modified Stejskal-Tanner diffusion encoding scheme, permitting a marked reduction in TE, in diffusion-weighted imaging of stroke patients at 3 T. Invest Radiol 45(1):29-35. https://doi.org/10.1097/ rli.0b013e3181c65c11

56. Caruyer E, Lenglet C, Sapiro G et al (2013) Design of multishell sampling schemes with uniform coverage in diffusion MRI. Magn Reson Med 69(6):1534-1540. https://doi.org/10.1002/mrm.24736

57. Jones DK, Williams Steve Charles, Rees Gasston D et al (2002) Isotropic resolution diffusion tensor imaging with whole brain acquisition in a clinically acceptable time. Hum Brain Mapp 15(4):216-230

58. Jenkinson M, Bannister P, Brady M et al (2002) Improved optimization for the robust and accurate linear registration and motion correction of brain images. Neuroimage 17(2):825-841

59. Jenkinson M, Beckmann CF, Behrens TEJ et al (2012) Fsl. Neuroimage 62(2):782-790. https://doi.org/10.1016/j.neuroimage .2011 .09 .015

60. Oishi K (2011) MRI atlas of human white matter, 2nd edn. Elsevier/Academic Press, Boston

61. Cho Z-H (2010) 7.0 Tesla MRI brain atlas. Springer, New York

62. Heidemann RM, Anwander A, Feiweier T et al. Sub-millimeter diffusion MRI at 7 T: Does resolution matter? In: ISMRM 20th Annual Meeting, Melbourne, 2012

63. Kleinnijenhuis M, van Mourik T, Norris DG et al (2015) Diffusion tensor characteristics of gyrencephaly using high resolution diffusion MRI in vivo at 7 T. Neuroimage 109:378-387. https:// doi.org/10.1016/j.neuroimage.2015.01.001

64. Toga AW, Thompson PM, Mori S et al (2006) Towards multimodal atlases of the human brain. Nat Rev Neurosci 7(12):952966. https://doi.org/10.1038/nrn2012

65. Basser PJ, Jones DK (2002) Diffusion-tensor MRI: theory, experimental design and data analysis - a technical review. NMR Biomed 15(7-8):456-467. https://doi.org/10.1002/nbm.783

66. Chung J-Y, In M-H, Oh S-H et al (2011) An improved PSF mapping method for EPI distortion correction in human brain at ultra high field (7 T). MAGMA 24(3):179-190. https://doi.org/10.1007/ s10334-011-0251-1

67. Feinberg DA, Setsompop K (2013) Ultra-fast MRI of the human brain with simultaneous multi-slice imaging. J Magn Reson 229:90-100. https://doi.org/10.1016/j.jmr.2013.02.002

68. Deistung A, Schafer A, Schweser F et al (2013) High-resolution MR imaging of the human brainstem in vivo at 7 Tesla. Front Hum Neurosci 7:710. https://doi.org/10.3389/fnhum.2013.00710

69. Yin Z, Magin RL, Klatt D (2014) Simultaneous MR elastography and diffusion acquisitions: diffusion-MRE (dMRE). Magn Reson Med 71(5):1682-1688. https://doi.org/10.1002/mrm.25180

70. Jeurissen B, Tournier J-D, Dhollander T et al (2014) Multi-tissue constrained spherical deconvolution for improved analysis of multi-shell diffusion MRI data. Neuroimage 103:411-426. https ://doi.org/10.1016/j.neuroimage.2014.07.061

71. Dyrby TB, Lundell H, Burke MW et al (2014) Interpolation of diffusion weighted imaging datasets. Neuroimage 103:202-213. https://doi.org/10.1016/j.neuroimage.2014.09.005 